\title{
HEAT FLOW FOR HORIZONTAL HARMONIC MAPS INTO A CLASS OF CARNOT-CARATHEODORY SPACES
}

\author{
JÜRgen Jost AND YI-Hu YANG
}

\section{Introduction}

Let $X$ and $B$ be two Riemannian manifolds with $\pi: X \rightarrow B$ being a Riemannian submersion. Let $\mathcal{H}$ be the corresponding horizontal distribution, which is perpendicular to the tangent bundle of the fibres of $\pi$. Then $X$ (just considered as a differentiable manifold), together with the distribution $\mathcal{H}$, forms a so-called Carnot-Caratheodory space (for details, see [1]), when the Riemannian metric of $X$ is restricted to $\mathcal{H}$. Considering $X$ as a Carnot-Caratheodory space, one can then define the notions of Carnot-Caratheodory distance (sometimes called subRiemannian distance), (minimizing) geodesic, completeness (under the CarnotCaratheodory distance), etc; a geodesic is actually a horizontal curve which locally realizes the Carnot-Caratheodory distance. In this note, we always assume that $X$ is complete, as both a Riemannian manifold and a Carnot-Caratheodory space; and the Riemannian submersion $\pi: X \rightarrow B$ together with its horizontal distribution $\mathcal{H}$ satisfies the following conditions

Condition 1: The Chow condition: the vector fields of $\mathcal{H}, X_{1}, X_{2}, \cdots$, and their iterated Lie brackets $\left[X_{i}, X_{j}\right],\left[\left[X_{i}, X_{j}\right], X_{k}\right], \cdots$ span the tangent space $T_{x} X$ at every point of $X$.

Condition 2: The sectional curvature of $X$ (as a Riemannian manifold) is nonpositive in the horizontal distribution $\mathcal{H}$.

Remarks. 1) The Chow condition guarantees that one has the so-called HopfRinow theorem (cf. [1]): if $X$ is complete under the Carnot-Caratheodory metric, then any two points can be joined by a minimizing geodesic (under the CarnotCaratheodory distance); moreover, in any given homotopic class of horizontal curves connecting two points, there exists a minimizing geodesic (under the Carnot-Caratheodory distance) connecting these points. 2) The Riemannian length of a horizontal curve is equal to the Carnot-Caratheodory length by the definitions.

In the sequel, unless stated otherwise, we always assume that the Riemannian submersion $\pi: X \rightarrow B$ in question satisfies the above conditions.

Received by the editors September 29, 2003.

The second named author supported partially by NSF of China (No. 10171077, 10471105) and "Shuguang Project" of the Committee of Education of Shanghai (04SG21). 
Our interest in this note is to study horizontal maps from a compact Riemannian manifold $M$ into a class of Carnot-Caratheodory spaces $(X, \mathcal{H})$ (see $\S 2)$, i.e. the image of the derivative of such a map lies in $\mathcal{H}$. We wish to find such maps that furthermore satisfy some additional differential equation, like the harmonic map equation, when $X$ is considered as a Riemannian manifold. We call a map horizontal harmonic if it is both horizontal and harmonic.

We have the space of smooth maps from $M$ into $\pi: X \rightarrow B$ that are horizontal and horizontally homotopic to a fixed horizontal map $g$, denoted by $B_{g, \mathcal{H}}^{\circ}(M ; X)$. It is easy to see that, under a certain suitable metric (defined by using some suitable Sobolev norm $), B_{g, \mathcal{H}}^{\circ}(M ; X)$ can be completed into a Banach manifold, denoted by $B_{g, \mathcal{H}}(M ; X)$, which is obviously an infinite dimensional manifold. Clearly, its tangent vectors are just horizontal vector fields of $X$ (more precisely, they are sections of a certain pull-back bundle). Similarly, considering the space of all maps from $M$ into $X$ (which are not necessarily horizontal), one can get another Banach manifold, denoted by $B(M ; X)$; and then $B_{g, \mathcal{H}}(M ; X)$ can be seen as a sub-manifold of $B(M ; X)$. It should be pointed out that these Banach manifolds may not be connected; it is however clear that they are locally connected; this is enough for our following discussion.

Corresponding to the horizontal distribution $\mathcal{H}$, one has an orthogonal projection from the tangent space of $B(M ; X)$ to that of $B_{g, \mathcal{H}}(M ; X)$, still denoted by $\mathcal{H}$. Given a vector field $\mathcal{X}$ of $B(M ; X)$ along $B_{g, \mathcal{H}}(M ; X)$, one can then define the orthogonal projection of $\mathcal{X}$, denoted by $\mathcal{H} \mathcal{X}$, which is a vector field of $B_{g, \mathcal{H}}(M ; X)$ and the value of which at any point of $B_{g, \mathcal{H}}(M ; X)$ is actually a horizontal vector field of $X$ (again, it is a section of a certain pull-back bundle).

In this note, we first give some examples of Carnot-Caratheodory spaces in which we are really interested. These spaces are actually a class of (locally) complex homogeneous manifolds which fibre over the corresponding symmetric spaces of noncompact type and the fiberations are Riemannian submersions under the standard invariant metrics. We will show that this class of spaces satisfy the Conditions 1) and 2) above. On the other hand, this class of homogeneous spaces, as Riemannian manifolds, are clearly complete, so this class of homogeneous spaces, as Carnot-Caratheodory spaces, are also complete under the corresponding Carnot-Caratheodory distance, since by the definition of Carnot-Caratheodory distance the Riemannian distance is not greater than the Carnot-Caratheodory distance. Thus we can apply the Banach spaces defined above to this class of homogeneous complex manifolds.

We next consider the following heat flow from $M \times[0, \infty)$ into $\pi: X \rightarrow B$

$$
\text { (*) } \mathcal{H} \tau(u(\cdot, t))-\frac{\partial u(\cdot, t)}{\partial t}=0,
$$

with the initial data $u(\cdot, 0)=g(\cdot)$, where $\tau(u(\cdot, t))$ is the usual tension field of $u(\cdot, t): M \rightarrow X, g(\cdot)$ is a smooth horizontal map. We show that one can always deform horizontally any smooth horizontal map into a horizontal harmonic map. It is worth noting that the operator $\mathcal{H} \tau$, as applied to the Banach space $B(M ; X)$, 
is not elliptic in general, but if applied to the Banach space $B_{g, \mathcal{H}}(M ; X)$, it is indeed elliptic, i.e. the symbol of its linearization is an isomorphism from the horizontal tangent subbundle of $X$ to itself, and hence one can apply the implicit function theorem to the Banach space $B_{g, \mathcal{H}}(M ; X)$ to obtain the short-time existence of a solution of $(*)$ with initial map $g$.

\section{A class of Carnot-Caratheodory spaces}

In this section, we will show some concrete examples for Carnot-Caratheodory spaces, which are actually the objects in which we are really interested. These examples are a class of (locally) complex homogeneous manifolds [3, 6]: Let $G$ be a connected noncompact real semi-simple Lie group with a compact Cartan subgroup; this means that if $K$ is a maximal compact subgroup of $G$, then $G$ and $K$ have the same rank; we also assume that $G / K$ is not a Hermitian symmetric space, although the case of Hermitian symmetric spaces also satisfies the previous property - this is equivalent to saying that the homogenous complex manifolds in question are nontrivial fiberation spaces over certain symmetric spaces of noncompact type, i.e. they have nontrivial vertical fibers, as seen below. Denoting such a compact Cartan subgroup by $H$ and choosing a suitable circle subgroup $T$ of $H$, we can then consider the centralizer $Z$ in $G$ of the circle subgroup $T$, which we know by Lie theory is contained in the maximal compact subgroup $K$. Taking the quotients $G / Z$ and $G / K, G / Z$ then is a homogeneous complex manifold [7] and can be considered as a fibration on the symmetric space $G / K$ of noncompact type with the fiber $K / Z$. Furthermore, under the standard invariant metrics, the fibration $\pi: G / K \rightarrow G / Z$ is a Riemannian submersion, and hence it has a horizontal distribution, denoted by $\mathcal{H}$, which satisfies all the assumptions mentioned in the Introduction, as will be shown in the following propositions.

Let $\Gamma$ be a discrete subgroup of $G$. Due to the discreteness of $\Gamma$ and the compactness of $K$, one can assume that $\Gamma \cap K=\emptyset$. Thus by taking quotients by $\Gamma$ we also have the Riemannian submersion $\Gamma \backslash G / Z \rightarrow \Gamma \backslash G / K$. Similarly, one also has a horizontal distribution, which is just the quotient by $\Gamma$ of the above $\mathcal{H}$, denoted by $\mathcal{H}^{\prime}$, and hence also satisfies the assumptions in the Introduction.

By the Cartan classification theorem for simple groups [4], the simple Lie groups $G$ satisfying the conditions stated in the first paragraph of this section can be listed as follows :

$$
\begin{array}{cl}
\mathrm{SO}(p, 2 q) q \geq 2 & \mathbf{e}_{8(8)} \\
\mathrm{S} p(p, q) & \mathbf{e}_{8(-24)} \\
\mathbf{e}_{6(2)} & \mathbf{f}_{4(4)} \\
\mathbf{e}_{7(7)} & \mathbf{f}_{4(-20)} \\
\mathbf{e}_{7(-5)} & \mathbf{g}_{2(2)}
\end{array}
$$

These groups are called groups of Hodge type but not of Hermitian type in Simpson's paper [10]. 
In the remaining part of this section, we will show that the distribution $\mathcal{H}$ and hence $\mathcal{H}^{\prime}$ do satisfy those assumptions. First, we check the assumption for sectional curvature in the horizontal direction $\mathcal{H}$. In fact, one has the following general

Proposition 1. Let $\pi: X \rightarrow B$ be a Riemannian submersion. If $B$ has nonpositive sectional curvature, then $X$ also has non-positive sectional curvature in the horizontal direction $\mathcal{H}$.

Remark. Since $G / K$ is a symmetric space of noncompact type, so it has nonpositive sectional curvature. Therefore by the proposition $G / Z$ also has nonpositive sectional curvature in the horizontal direction $\mathcal{H}$,

Proof of Proposition 1. The proof is a simple consequence of the O'Neill formulae [8]. To this end, we first recall an idea of B. O'Neill $[2,8]$ : One can define a type $(2,1)$-tensor field on $X$, denoted by $A$, as follows: for any two vector fields $Y, Z$ on $X$,

$$
A(Y, Z)=\mathcal{H} \nabla_{\mathcal{H} Y} \mathcal{V} Z+\mathcal{V} \nabla_{\mathcal{H} Y} \mathcal{H} Z
$$

where $\mathcal{H}$ and $\mathcal{V}$ mean taking the horizontal part and the vertical part respectively, and $\nabla$ is the Riemannian connection of $X$. An easy calculation shows that $A$ indeed is a tensor field on $X$, namely, the value of $A(Y, Z)$ at any fixed point $x$ depends only on the values of $Y$ and $Z$ at $x$, although its definition does depend on the values of $Y$ and $Z$ on a small neighborhood of $x$. Moreover, it has the following key property (here we state slightly more than we need):

$$
A(Y, Z)=-A(Z, Y)=\frac{1}{2} \mathcal{V}[Y, Z]
$$

for any two horizontal vectors $Y$ and $Z$. The proof of the property is simple: It is sufficient to show $A(Y, Y)=0$, since if it is the case, $A(Y+Z, Y+Z)=$ $A(Y, Z)+A(Z, Y)=0$; and, by the definition of $A$,

$$
\mathcal{V}[Y, Z]=\mathcal{V} \nabla_{Y} Z-\mathcal{V} \nabla_{Z} Y=A(Y, Z)-A(Z, Y) .
$$

Since $A$ is a tensor, one can take the horizontal vector field $Y$ being the unique lift of a vector field $Y^{\prime}$ on $B$, i.e. $\pi_{*}(Y)=Y^{\prime}$. Let $U$ be any vertical vector field on $X$. Then we have $\pi_{*}[Y, U]=\left[\pi_{*} Y, \pi_{*} U\right]=0$ since $[Y, U]$ is a vertical vector field on $X$. Thus one has, by the torsion-freeness of the connection $\nabla$,

$$
\begin{gathered}
<A(Y, Y), U>=<\nabla_{Y} Y, U>=-<Y, \nabla_{Y} U> \\
=-<Y,[Y, U]+\nabla_{U} Y>=-<Y, \nabla_{U} Y>=-\frac{1}{2} U|Y|^{2} .
\end{gathered}
$$

Since $Y$ is the lift of a vector field $Y$ of $B$, so $|Y|^{2}$ is constant on any fiber of $\pi: X \rightarrow B$, and hence $\langle A(Y, Y), U\rangle=0$ for any vertical vector $U$. On the other hand, by the definition, $A(Y, Y)$ is a vertical vector, so $A(Y, Y)=0$.

We now turn to the proof of the proposition. Denoting the curvature tensors of $X$ and $B$ by $R$ and $R^{\prime}$ respectively, one of the O'Neill formulae then says, for 
horizontal tangent vectors $Y, Z, U, V$ of $X$,

$$
\begin{aligned}
& <R(Y, Z) U, V>=<R^{\prime}(Y, Z) U, V>-2<A(Y, Z), A(U, V)> \\
& +<A(Z, U), A(Y, V)>-<A(Y, U), A(Z, V)>,
\end{aligned}
$$

where $Y, Z, U, V$ are also regarded as tangent vectors of $B$ by $\pi_{*}$. Since $A$ is skew-symmetric in the horizontal direction, so we have

$$
\begin{aligned}
& <R(Y, Z) Y, Z>=<R^{\prime}(Y, Z) Y, Z>-2<A(Y, Z), A(Y, Z)>+ \\
& <A(Z, Y), A(Y, Z)>=<R^{\prime}(Y, Z) Y, Z>-3<A(Y, Z), A(Y, Z)>\leq 0 .
\end{aligned}
$$

This finishes the proof of the proposition.

In order to show the Chow condition, we can turn the problem into a Lietheoretic one. To this end, we first need to give the relation between the Lie bracket of left invariant vector fields and the Lie bracket of the Lie algebras in question when considering left invariant vector fields as elements of the Lie algebra. We always use the notations of [7]. Denoting the Lie algebra of $G$ and $Z$ by $\mathfrak{g}$ and $\mathfrak{z}$ respectively, it is easy to see that we have a direct sum decomposition of vector spaces

$$
\mathfrak{g}=\mathfrak{z}+\mathfrak{m}
$$

with $[\mathfrak{z}, \mathfrak{m}] \subset \mathfrak{m}$. Here $\mathfrak{m}$ can be identified with the tangent space of $G / Z$ at the origin or the set of all $G$-invariant vector fields on $G / Z$. Theorem 2.10 of [7] tells us that there exists a unique torsion-free $G$-invariant affine connection $\nabla$ with

$$
\nabla_{Y} Z=\frac{1}{2}[Y, Z]_{\mathfrak{m}}, \text { for } Y, Z \in \mathfrak{m},
$$

here by $Y, Z$ on the left-hand side we mean vector fields on $G / Z$ while $Y, Z$ on the right-hand side mean elements in $\mathfrak{g} ;[Y, Z]_{\mathfrak{m}}$ denotes the $\mathfrak{m}$-component of $[Y, Z]$. Thus, one has

$$
[Y, Z]=[Y, Z]_{\mathfrak{m}},
$$

here by the left-hand side we mean the Lie bracket of vector fields, afterwards we will not point out this since it should be clear from the context. As before, one has a Cartan subgroup $H$ contained in $Z$, the Lie algebra of which is a maximal Abelian subalgebra, denoted by $\mathfrak{h}$. Consider the Cartan decomposition $\mathfrak{g}=\mathfrak{k}+\mathfrak{p}$, here $\mathfrak{k}$ is the Lie algebra of $K$. We then have the relations $\mathfrak{h} \subset \mathfrak{z} \subset \mathfrak{k} \subset \mathfrak{g}$ and $\mathfrak{p} \subset \mathfrak{m}$. Again, $\mathfrak{p}$, as a vector subspace of $\mathfrak{m}$, can be identified with the horizontal tangent subspace at the origin with respect to the Riemannain submersion $G / Z \rightarrow G / K$ and its left translation forms the horizontal distribution $\mathcal{H}$ of the Riemannian submersion; furthermore, its elements can be identified with $G$-invariant horizontal vector fields of $G / K$. By the previous relation of the two Lie brackets, in order to show that the horizontal distribution $\mathcal{H}$ satisfies the Chow condition, it is sufficient to show that $\mathfrak{p}$ and $[\mathfrak{p}, \mathfrak{p}]$ span $\mathfrak{m}$. To this end, we use the root system of the complexification $\mathfrak{g}^{\mathbf{C}}$ of $\mathfrak{g}$ correspoding to the Cartan subalgebra $\mathfrak{h}$. 
Let $\Delta$ be the root system of $\mathfrak{g}^{\mathbf{C}}$ with respect to $\mathfrak{h}, \mathfrak{g}^{\alpha}$ the root space corresponding to $\alpha \in \Delta, \mathfrak{g}^{\mathbf{C}}=\mathfrak{k}^{\mathbf{C}}+\mathfrak{p}^{\mathbf{C}}$ the Cartan decomposition, $\theta$ the Cartan involution, $\sigma$ the conjugation of $\mathfrak{g}^{\mathbf{C}}$ with respect to $\mathfrak{g}$. Since $\mathfrak{h}$ lies in $\mathfrak{k}$ while $[\mathfrak{k}, \mathfrak{k}] \subset \mathfrak{k}$ and $[\mathfrak{k}, \mathfrak{p}] \subset \mathfrak{p}$, so the root space $\mathfrak{g}^{\alpha}$ lies in either $\mathfrak{k}^{\mathbf{C}}$ or $\mathfrak{p}^{\mathbf{C}}$. In the first case, we call $\alpha$ a compact root, and we denote the set of all compact roots by $\Delta(\mathfrak{k})$; in the last case, it is a noncompact root, and we denote the set of noncompact roots by $\Delta(\mathfrak{p})$. On the other hand, we also have the direct sum decomposition for vector spaces $\mathfrak{g}=\mathfrak{h}+\mathfrak{m}^{\prime}$, obviously $\mathfrak{m} \subset \mathfrak{m}^{\prime}$; furthermore one has the direct $\operatorname{sum} \mathfrak{m}^{\prime}=\mathfrak{k}^{\prime}+\mathfrak{p}$ with $\mathfrak{h}+\mathfrak{k}^{\prime}=\mathfrak{k}$. So if we can show that $[\mathfrak{p}, \mathfrak{p}]=\mathfrak{k}^{\prime}$, equivalently $\left[\mathfrak{p}^{\mathbf{C}}, \mathfrak{p}^{\mathbf{C}}\right]=\mathfrak{k}^{\prime} \mathbf{C}$, then the Chow condition is obtained. From the root theory, we has

$$
\mathfrak{k}^{\prime \mathbf{C}}=\sum_{\alpha \in \Delta(\mathfrak{k})} \mathfrak{g}^{\alpha} \text { and } \mathfrak{p}^{\mathbf{C}}=\sum_{\alpha \in \Delta(\mathfrak{p})} \mathfrak{g}^{\alpha} .
$$

Note that $\sigma\left(\mathfrak{g}^{\alpha}\right)=\mathfrak{g}^{-\alpha}$ while $\sigma\left(\mathfrak{k}^{\prime} \mathbf{C}\right)=\mathfrak{k}^{\prime \mathbf{C}}$ and $\sigma\left(\mathfrak{p}^{\mathbf{C}}\right)=\mathfrak{p}^{\mathbf{C}}$, so if $\alpha \in \Delta(\mathfrak{k})$ (resp. $\Delta(\mathfrak{p}))$, then so is $-\alpha$. We now state the following

Proposition 2. For any root $\alpha \in \Delta(\mathfrak{k})$, there exist two noncompact roots $\beta$ and $\gamma$ with $\beta+\gamma=\alpha$.

Remark. Clearly if the proposition is true, then the Chow condition is obtained. In the following, we will case by case write down compact roots and noncompact roots of $\mathfrak{g}^{\mathbf{C}}$ for the above simple groups list and then easily check that the above assertion is true. We believe that the proposition should also have a general Lie-theoretic proof.

$\mathbf{S O}(\mathbf{p}, \mathbf{2 q}), \mathbf{q} \geq \mathbf{2}$ : Here, we have two cases to consider. The first is $S O(2 p, 2 q)$, $p, q \geq 2$ : It is the noncompact real form of $S O(2(p+q), \mathbf{C})$ with the maximal compact subgroup $K=S O(2 p) \times S O(2 q)$. The root system of $\mathfrak{s o}(2(p+q), \mathbf{C})$ is $D_{p+q}=\left\{ \pm e_{i} \pm e_{j}, 1 \leq i<j \leq p+q\right\}$, here $\left\{e_{i}\right\}$ is the standard basis of $\mathbf{R}^{p+q}$, while the root systems of $\mathfrak{s o}(2 p, \mathbf{C})$ and $\mathfrak{s o}(2 q, \mathbf{C})$, embedded in $D_{p+q}$, are

$$
D_{p}=\left\{ \pm e_{i} \pm e_{j}, 1 \leq i<j \leq p\right\}
$$

and

$$
D_{q}=\left\{ \pm e_{i} \pm e_{j}, p+1 \leq i<j \leq p+q\right\}
$$

respectively. Therefore, corresponding to the noncompact real form $S O(2 p, 2 q)$ and its compact Cartan subalgebra, $\mathfrak{s o}(2(p+q), \mathbf{C})$ has noncompact roots

$$
\left\{ \pm e_{i} \pm e_{j}, 1 \leq i \leq p, p+1 \leq j \leq p+q\right\} \text {. }
$$

The second case is $S O(2 p+1,2 q), p, q \geq 2$ : It is the noncompact real form of $S O(2(p+q)+1, \mathbf{C})$ with the maximal compact subgroup $K=S O(2 p+1) \times$ $S O(2 q)$. The root system of $\mathfrak{s o}(2(p+q)+1, \mathbf{C})$ is $B_{p+q}=\left\{ \pm e_{i}, \pm e_{i} \pm e_{j}, 1 \leq i, j \leq\right.$ $p+q, i \neq j\}$ while the root systems of $\mathfrak{s o}(2 p+1, \mathbf{C})$ and $\mathfrak{s o}(2 q, \mathbf{C})$, embedded in $B_{p+q}$, are

$$
B_{p}=\left\{ \pm e_{i}, \pm e_{i} \pm e_{j}, 1 \leq i, j \leq p, i \neq j\right\}
$$


and

$$
D_{q}=\left\{ \pm e_{i} \pm e_{j}, p+1 \leq i<j \leq p+q\right\}
$$

respectively. Therefore, corresponding to the noncompact real form $S O(2 p+1,2 q)$ and its compact Cartan subalgebra, $\mathfrak{s o}(2(p+q)+1, \mathbf{C})$ has noncompact roots

$$
\left\{ \pm e_{i} \pm e_{j}, \pm e_{j}, 1 \leq i \leq p, p+1 \leq j \leq p+q\right\}
$$

$\mathbf{S p}(\mathbf{p}, \mathbf{q})$ : It is the noncompact real form of $S p(p+q, \mathbf{C})$ with the maximal compact subgroup $K=S p(p) \times S p(q)$. The root system of $\mathfrak{s p}(p+q, \mathbf{C})$ is $C_{p+q}=\left\{ \pm 2 e_{i}, \pm e_{i} \pm e_{j}, 1 \leq i, j \leq p+q, i \neq j\right\}$, while the root systems of $\mathfrak{s p}(p, \mathbf{C})$ and $\mathfrak{s p}(q, \mathbf{C})$, embedded in $C_{p+q}$, are

$$
C_{p}=\left\{ \pm 2 e_{i}, \pm e_{i} \pm e_{j}, 1 \leq i, j \leq p, i \neq j\right\}
$$

and

$$
C_{q}=\left\{ \pm 2 e_{i}, \pm e_{i} \pm e_{j}, p+1 \leq i, j \leq p+q, i \neq j\right\}
$$

respectively; therefore, corresponding to the noncompact real form $S p(p, q)$ and its compact Cartan subalgebra, $\mathfrak{s p}(p+q, \mathbf{C})$ has noncompact roots

$$
\left\{ \pm e_{i} \pm e_{j}, 1 \leq i \leq p, p+1 \leq j \leq p+q\right\}
$$

$\mathbf{e}_{\mathbf{6}(2)}$ : It is the noncompact real form of $\mathbf{e}_{6}$ with the maximal compact subgroup $K=S U(6) \times S U(2)$. The root system of $\mathbf{e}_{6}$ is

$$
\begin{aligned}
E_{6}= & \left\{e_{i}-e_{j}, i \neq j, 1 \leq i, j \leq 6\right\} \cup\left\{ \pm\left(e_{7}-e_{8}\right)\right\} \cup \\
& \left\{\frac{1}{2}\left(e_{\sigma(1)}+e_{\sigma(2)}+e_{\sigma(3)}-e_{\sigma(4)}-e_{\sigma(5)}-e_{\sigma(6)} \pm\left(e_{7}-e_{8}\right)\right), \sigma \in P(6)\right\},
\end{aligned}
$$

where $P(6)$ is the permutation group of $\{1,2,3,4,5,6\}$. The root system of $\mathfrak{s l}(6, \mathbf{C})+\mathfrak{s l}(2, \mathbf{C})$, embedded in $E_{6}$, is

$$
A_{5}+A_{1}=\left\{e_{i}-e_{j}, i \neq j, 1 \leq i, j \leq 6\right\} \cup\left\{ \pm\left(e_{7}-e_{8}\right)\right\} .
$$

Thus, corresponding to the noncompact real form $\mathbf{e}_{6(2)}$ and its compact Cartan subalgebra, $\mathbf{e}_{6}$ has noncompact roots

$$
\left\{\frac{1}{2}\left(e_{\sigma(1)}+e_{\sigma(2)}+e_{\sigma(3)}-e_{\sigma(4)}-e_{\sigma(5)}-e_{\sigma(6)} \pm\left(e_{7}-e_{8}\right)\right), \sigma \in P(6)\right\} .
$$

$\mathbf{e}_{\mathbf{7}(\mathbf{7})}$ : It is the noncompact real form of $\mathbf{e}_{7}$ with the maximal compact subgroup $K=S U(8)$. The root system of $\mathbf{e}_{7}$ is

$$
\begin{aligned}
E_{7}= & \left\{e_{i}-e_{j}, 1 \leq i, j \leq 8, i \neq j\right\} \cup \\
& \left\{\frac{1}{2}\left(e_{\sigma(1)}+e_{\sigma(2)}+e_{\sigma(3)}+e_{\sigma(4)}-e_{\sigma(5)}-e_{\sigma(6)}-e_{\sigma(7)}-e_{\sigma(8)}\right), \sigma \in P(8)\right\},
\end{aligned}
$$


here $P(8)$ is the permutation group of $\{1,2,3,4,5,6,7,8\}$. The root system of $\mathfrak{s l}(8, \mathbf{C})$, embedded in $E_{7}$, is

$$
A_{7}=\left\{e_{i}-e_{j}, i \neq j, 1 \leq i, j \leq 8\right\} .
$$

Thus, corresponding to the noncompact real form $\mathbf{e}_{7(7)}$ and its compact Cartan subalgebra, $\mathbf{e}_{7}$ has noncompact roots

$$
\left\{\frac{1}{2}\left(e_{\sigma(1)}+e_{\sigma(2)}+e_{\sigma(3)}+e_{\sigma(4)}-e_{\sigma(5)}-e_{\sigma(6)}-e_{\sigma(7)}-e_{\sigma(8)}\right), \sigma \in P(8)\right\} .
$$

$\mathbf{e}_{\mathbf{7}(-\mathbf{5})}$ : It is the noncompact real form of $\mathbf{e}_{7}$ with the maximal compact subgroup $K=S O(12) \times S U(2)$. The root system of $\mathbf{e}_{7}$ is

$$
\begin{aligned}
E_{7}= & \left\{e_{i}-e_{j}, 1 \leq i, j \leq 8, i \neq j\right\} \cup \\
& \left\{\frac{1}{2}\left(e_{\sigma(1)}+e_{\sigma(2)}+e_{\sigma(3)}+e_{\sigma(4)}-e_{\sigma(5)}-e_{\sigma(6)}-e_{\sigma(7)}-e_{\sigma(8)}\right), \sigma \in P(8)\right\},
\end{aligned}
$$

here $P(8)$ is the permutation group of $\{1,2,3,4,5,6,7,8\}$. The root system of $\mathfrak{s o}(12, \mathbf{C})+\mathfrak{s l}(2, \mathbf{C})$, embedded in $E_{7}$, is

$$
\begin{aligned}
D_{6}+A_{1}= & \left\{e_{i}-e_{j}, 1 \leq i, j \leq 6, i \neq j\right\} \cup \\
& \left\{ \pm \frac{1}{2}\left(e_{\sigma(1)}+e_{\sigma(2)}+e_{\sigma(3)}+e_{\sigma(4)}-e_{\sigma(5)}-e_{\sigma(6)}-e_{7}-e_{8}\right)\right\} \cup \\
& \left\{ \pm\left(e_{7}-e_{8}\right)\right\} .
\end{aligned}
$$

(Note that if letting $\mathbf{R}^{n}$ have the standard basis $\left\{f_{1}, \cdots f_{n}\right\}, D_{n}=\left\{ \pm f_{i} \pm f_{j}, i \neq\right.$ $j\}$; so we need to construct an isomorphism between $D_{6}$ and $\left\{e_{i}-e_{j}, 1 \leq i, j \leq\right.$ $6, i \neq j\} \cup\left\{ \pm \frac{1}{2}\left(e_{\sigma(1)}+e_{\sigma(2)}+e_{\sigma(3)}+e_{\sigma(4)}-e_{\sigma(5)}-e_{\sigma(6)}-e_{7}-e_{8}\right)\right\}$. This is done by the uniqueness: $\left\{e_{i}-e_{j}, 1 \leq i, j \leq 6, i \neq j\right\} \cup\left\{ \pm \frac{1}{2}\left(e_{\sigma(1)}+e_{\sigma(2)}+\right.\right.$ $\left.\left.e_{\sigma(3)}+e_{\sigma(4)}-e_{\sigma(5)}-e_{\sigma(6)}-e_{7}-e_{8}\right)\right\}$ indeed is a root system of cardinality $60 ;$ on the other hand, the only root system of cardinality 60 is $D_{n}$ by the Cartan classification theorem.)

Therefore, corresponding to the noncompact real form $\mathbf{e}_{7(-5)}$ and its compact Cartan subalgebra, $\mathbf{e}_{7}$ has noncompact roots

$$
\begin{aligned}
& \left\{ \pm\left(e_{i}-e_{7}\right), \pm\left(e_{i}-e_{8}\right), 1 \leq i \leq 6\right\} \cup \\
& \left\{ \pm \frac{1}{2}\left(e_{\sigma(1)}+e_{\sigma(2)}+e_{\sigma(3)}-e_{\sigma(4)}-e_{\sigma(5)}-e_{\sigma(6)}+e_{7}-e_{8}\right)\right\} .
\end{aligned}
$$

$\mathbf{e}_{\mathbf{8}(\mathbf{8})}$ : It is the noncompact real form of $\mathbf{e}_{8}$ with the maximal compact subgroup $K=S O(16)$. The root system of $\mathbf{e}_{8}$ is

$$
E_{8}=\left\{ \pm e_{i} \pm e_{j}, \frac{1}{2} \sum_{i=1}^{8}(-1)^{m(i)} e_{i} \text { with } \sum m(i) \text { being even, } 1 \leq i, j \leq 8\right\}
$$

where $m(i)$ is 0 or 1 . The root system of $\mathfrak{s o}(16, \mathbf{C})$, embedded in $E_{8}$, is $D_{8}=$ $\left\{ \pm e_{i} \pm e_{j}, 1 \leq i, j \leq 8\right\}$. Therefore, corresponding to the noncompact real form 
$\mathbf{e}_{8(8)}$ and its compact Cartan subalgebra, $\mathbf{e}_{8}$ has noncompact roots

$$
\left\{\frac{1}{2} \sum_{i=1}^{8}(-1)^{m(i)} e_{i} \text { with } \sum m(i) \text { being even, } 1 \leq i, j \leq 8\right\} .
$$

$\mathbf{e}_{\mathbf{8}(-\mathbf{2 4})}$ : It is the noncompact real form of $\mathbf{e}_{8}$ with the maximal compact subgroup $K=\mathbf{e}_{7(-133)} \times S U(2)$. The root system of $\mathbf{e}_{8}$ is

$$
E_{8}=\left\{ \pm e_{i} \pm e_{j}, \frac{1}{2} \sum_{i=1}^{8}(-1)^{m(i)} e_{i} \text { with } \sum m(i) \text { being even, } 1 \leq i, j \leq 8\right\}
$$

The root system of $\mathbf{e}_{7}+\operatorname{sl}(2, \mathbf{C})$, embedded in $E_{8}$, is

$$
\begin{aligned}
& E_{7}+A_{1}=\left\{e_{i}-e_{j}, 1 \leq i, j \leq 8, i \neq j\right\} \cup \\
& \left\{\frac{1}{2}\left(e_{\sigma(1)}+e_{\sigma(2)}+e_{\sigma(3)}+e_{\sigma(4)}-e_{\sigma(5)}-e_{\sigma(6)}-e_{\sigma(7)}-e_{\sigma(8)}\right), \sigma \in P(8)\right\} \\
& \cup\left\{ \pm \frac{1}{2}\left(e_{1}+e_{2}+\cdots+e_{8}\right)\right\} .
\end{aligned}
$$

Thus, corresponding to the noncompact real form $\mathbf{e}_{8(-24)}$ and its compact Cartan subalgebra, $\mathbf{e}_{8}$ has noncompact roots

$$
\begin{aligned}
& \left\{ \pm\left(e_{i}+e_{j}\right), 1 \leq i<j \leq 8\right\} \cup \\
& \left\{\frac{1}{2}\left(e_{\sigma(1)}+e_{\sigma(2)}+e_{\sigma(3)}+e_{\sigma(4)}+e_{\sigma(5)}+e_{\sigma(6)}-e_{\sigma(7)}-e_{\sigma(8)}\right), \sigma \in P(8)\right\} .
\end{aligned}
$$

$\mathbf{f}_{\mathbf{4}(\mathbf{4})}$ : It is the noncompact real form of $\mathbf{f}_{4}$ with the maximal compact subgroup $S p(3) \times S U(2)$. The root system of $\mathbf{f}_{4}$ is

$$
F_{4}=\left\{ \pm e_{i}, \pm e_{i} \pm e_{j}(1 \leq i, j \leq 4, i \neq j), \frac{1}{2}\left( \pm e_{1} \pm e_{2} \pm e_{3} \pm e_{4}\right)\right\}
$$

while the root system of $\mathfrak{s p}(3, \mathbf{C})+\mathfrak{s l}(2, \mathbf{C})$, embedded in $F_{4}$, is

$$
C_{3}+A_{1}=\left\{ \pm 2 f_{i}, \pm f_{i} \pm f_{j}, 1 \leq i, j \leq 3, i \neq j\right\} \cup\left\{ \pm\left(e_{3}+e_{4}\right)\right\}
$$

where $f_{1}=\frac{1}{2}\left(e_{1}-e_{2}\right), f_{2}=\frac{1}{2}\left(e_{1}+e_{2}\right), f_{3}=\frac{1}{2}\left(e_{3}-e_{4}\right)$. Thus, corresponding to the noncompact real form $\mathbf{f}_{4(4)}$ and its compact Cartan subalgebra, $\mathbf{f}_{4}$ has noncompact roots

$$
\begin{aligned}
& \left\{ \pm e_{3}, \pm e_{4}, \pm e_{i} \pm e_{j}, i=1,2, j=3,4\right\} \cup \\
& \left\{\frac{1}{2}\left( \pm\left(e_{1}-e_{2}\right) \pm\left(e_{3}+e_{4}\right)\right), \frac{1}{2}\left( \pm\left(e_{1}+e_{2}\right) \pm\left(e_{3}+e_{4}\right)\right)\right\}
\end{aligned}
$$

$\mathbf{f}_{\mathbf{4}(-\mathbf{2 0})}$ : It is the noncompact real form of $\mathbf{f}_{4}$ with the maximal compact subgroup $S O(9)$. The root system of $\mathbf{f}_{4}$ is

$$
F_{4}=\left\{ \pm e_{i}, \pm e_{i} \pm e_{j}(1 \leq i, j \leq 4, i \neq j), \frac{1}{2}\left( \pm e_{1} \pm e_{2} \pm e_{3} \pm e_{4}\right)\right\}
$$


while the root system of $\mathfrak{s o}(9, \mathbf{C})$, embedded in $F_{4}$, is $B_{4}=\left\{ \pm e_{i}, \pm e_{i} \pm e_{j}, 1 \leq\right.$ $i, j \leq 4, i \neq j\}$. Therefore, corresponding to the noncompact real form $\mathbf{f}_{4(-20)}$ and its compact Cartan subalgebra, $\mathbf{f}_{4}$ has noncompact roots

$$
\left\{\frac{1}{2}\left( \pm e_{1} \pm e_{2} \pm e_{3} \pm e_{4}\right)\right\}
$$

$\mathbf{g}_{\mathbf{2}(\mathbf{2})}$ : It is the noncompact real form of $\mathbf{g}_{2}$ with the maximal compact subgroup $S U(2) \times S U(2)$. The root system of $\mathbf{g}_{2}$ is

$$
G_{2}=\{ \pm \alpha, \pm \beta, \pm(\alpha+\beta), \pm(2 \alpha+\beta), \pm(3 \alpha+\beta), \pm(3 \alpha+2 \beta)\}
$$

where $\alpha=e_{1}, \beta=-\frac{3}{2} e_{1}+\frac{\sqrt{3}}{2} e_{2}$; while the root system of $\mathfrak{s l}(2, \mathbf{C})+\mathfrak{s l}(2, \mathbf{C})$, embedded in $G_{2}$, is $A_{1}+A_{1}=\{ \pm \beta\} \bigcup\{ \pm(2 \alpha+\beta)\}$. Therefore the noncompact root system is

$$
\{ \pm \alpha, \pm(\alpha+\beta), \pm(3 \alpha+\beta), \pm(3 \alpha+2 \beta)\}
$$

Summing all the above up, it is easy to check that the noncompact roots can generate the compact roots, i.e. for any compact root $\alpha$ there exist two noncompact roots $\beta$ and $\gamma$ satisfying $\alpha=\beta+\gamma$.

\section{Heat flow for horizontal harmonic maps}

Let $\pi: X \rightarrow B$ be a Riemannian submersion, $\mathcal{H}$ the corresponding horizontal distribution, and $M$ a compact Riemannian manifold. Assume that $\pi: X \rightarrow B$ satisfies the conditions stated in the Introduction, i.e. the Chow condition and $B$ having non-positive sectional curvature, and assume that $X$ is complete under both the Carnot-Caratheodory distance and the Riemannian metric. Consider the following heat equation on $M$

$$
\text { (*) } \mathcal{H} \tau(u)-\frac{\partial u}{\partial t}=0
$$

where $\mathcal{H}$ represents the projection to $\mathcal{H}$, and $\tau$ is the tension field of $u$. Assume that $u$ has the initial data $u(\cdot, 0)=g(\cdot)$. We always assume that $g$ is a horizontal map from $M$ to $X$ and smooth. We wish to obtain some horizontal harmonic map from $M$ into $X$ by solving the above heat equation under the initial data $g$, when $X$ is considered as a Riemannian manifold.

Lemma 1. There exists a positive number $T$ with the property that the equation (*) with the initial data $g$ has a smooth solution $u(x, t)$ for $t \in[0, T)$ satisfying $u(\cdot, t) \in B_{g, \mathcal{H}}(M ; X)$. Furthermore, if $u(x, t)$ is a solution of $(*)$ with $u(\cdot, 0)=$ $g(\cdot)$ for $t \in\left[0, T^{\prime}\right), T^{\prime}>0$, then $u(\cdot, t) \in B_{g, \mathcal{H}}(M ; X)$ and hence $\frac{u(\cdot, t)}{\partial t}$ is a horizontal tangent vector field of $X$ for any $t \in\left[0, T^{\prime}\right)$.

Proof. The first part of the lemma is essentially a standard result if one restricts the problem to the space $B_{g, \mathcal{H}}(M ; X)$ : The symbol of the linearization of the operator $\mathcal{H} \tau$ is just an isomorphism from the horizontal tangent subbundle of $X$ to itself, so $\mathcal{H} \tau$ is elliptic. Thus one can still apply the implicit function 
theorem to the present case, as one applies the implicit function theorem to the usual harmonic map heat flow, to obtain the short-time existence. As for the second part, it is also easy to see from the following discussion. Since $\mathcal{H} \tau(u)$ is a horizontal vector on $B(M ; X)$, i.e. a horizontal vector field on $X$, so $\frac{\partial u}{\partial t}$ is also horizontal. Fix a point $x \in M$ and take arbitrarily a curve $\gamma(s)$ starting from $x$ for $s \in\left[0, s_{0}\right]$ and a vertical tangent vector $V$ at $g(x)$, translate parallelly $V$ along the $t$-curve $u(x, t)$ and then the $s$-curves $u(\gamma(s), t)$, still denoted by $V$. Note that $V$ is not necessarily parallel, even not continuous, along the $t$-curves $u(\gamma(s), t)$ for $s \neq 0$. Compute $\frac{\partial}{\partial t}<\frac{\partial}{\partial s} u(\gamma(0), t), V>$

$$
\begin{gathered}
\frac{\partial}{\partial t}<\frac{\partial}{\partial s} u(\gamma(0), t), V>=<\nabla_{\frac{\partial}{\partial t}} \frac{\partial}{\partial s} u(\gamma(0), t), V> \\
=<\nabla_{\frac{\partial}{\partial s}} \frac{\partial}{\partial t} u(\gamma(0), t), V>=\frac{\partial}{\partial s}<\frac{\partial}{\partial t} u(\gamma(0), t), V>=0 .
\end{gathered}
$$

Since $<\frac{\partial}{\partial s} u(\gamma(0), t), V>\left.\right|_{t=0}=<\frac{\partial}{\partial s} g(\gamma(0)), V>=0$, so $<\frac{\partial}{\partial s} u(\gamma(0), t), V>=$ 0 . Thus $u(\cdot, t)$ is horizontal. Then, the horizontality of $\frac{u(\cdot, t)}{\partial t}$ implies $u(\cdot, t) \in$ $B_{g, \mathcal{H}}(M ; X)$. The lemma is obtained.

Let $e(u)(x, t)=\frac{1}{2}|\nabla u|^{2}(x, t)$ be the energy density of $u(\cdot, t)$ for $t \in[0, T)$. Denote the Laplace operator of $M$ by $\Delta$ and take $\left\{e_{i}\right\}$ as a normal frame of $M$; denote the Ricci tensor of $M$ by $\operatorname{Ric}^{M}$ and the curvature tensor of $X$ by $R^{X}$. By $\mathcal{V}$ we mean taking the vertical components of vectors. Then computing $\left(\Delta-\frac{\partial}{\partial t}\right) e(u)$, we obtain

$$
\begin{aligned}
\left(\Delta-\frac{\partial}{\partial t}\right) e(u)= & <\nabla_{e_{i}} \nabla_{e_{i}} \mathrm{~d} u, \mathrm{~d} u>+|\nabla \mathrm{d} u|^{2}-<\nabla \frac{\partial u}{\partial t}, \mathrm{~d} u> \\
= & <\nabla(\mathcal{V} \tau(u)), \mathrm{d} u>+|\nabla \mathrm{d} u|^{2}+<\operatorname{Ric}^{M}\left(\mathrm{~d} u\left(e_{i}\right), \mathrm{d} u\left(e_{i}\right)\right)> \\
& -<R^{X}\left(\mathrm{~d} u\left(e_{i}\right), \mathrm{d} u\left(e_{j}\right)\right) \mathrm{d} u\left(e_{i}\right), \mathrm{d} u\left(e_{j}\right)> \\
= & -|\mathcal{V} \tau(u)|^{2}+|\nabla \mathrm{d} u|^{2}+<\operatorname{Ric}^{M}\left(\mathrm{~d} u\left(e_{i}\right), \mathrm{d} u\left(e_{i}\right)\right)> \\
& -<R^{X}\left(\mathrm{~d} u\left(e_{i}\right), \mathrm{d} u\left(e_{j}\right)\right) \mathrm{d} u\left(e_{i}\right), \mathrm{d} u\left(e_{j}\right)>.
\end{aligned}
$$

In the second equality above we used the Weitzenböck formula and the equation $(*)$; in the last equality we used the horizontality of $u$. The following observation is important for the present study.

Lemma 2. Let $\pi: X \rightarrow B$ be a Riemannian submersion. Then, for any horizontal map u from a Riemannian manifold $M$ into $X$, the vertical part $\mathcal{V} \tau(u)$ of its tension field $\tau(u)$ vanishes.

Remark. Since the horizontal distribution $\mathcal{H}$ is generally not integrable, so the vertical part of the Hessian of a horizontal map $u$ does not necessarily vanish.

Proof of Lemma 2. To prove Lemma 2, we need to use some arguments from the proof of Proposition 1, namely the definition of the tensor $A$ and its skewsymmetry. Take a normal frame $\left\{e_{i}\right\}$ of $M$ and an orthogonal frame of $X$ as follows: $\left\{e_{\alpha}, e_{\beta}, e_{\gamma}, \cdots, e_{\mu}, e_{\nu}, \cdots\right\}$ with the properties $\left\{e_{\alpha}, \cdots\right\}$ being horizontal 
and $\left\{e_{\mu}, \cdots\right\}$ vertical (note that, under such a restriction, one cannot get a normal frame in general). Then, under these frames, the tension field of the horizontal map $u$ can be written as

$$
\tau(u)=\sum_{i} \nabla \mathrm{d} u\left(e_{i}, e_{i}\right)=\sum_{i, \alpha} u_{i i}^{\alpha} e_{\alpha}+\sum_{i, \alpha, \beta} u_{i}^{\alpha} u_{i}^{\beta} \nabla_{e_{\beta}} e_{\alpha}
$$

and hence its vertical part is $\sum_{i, \alpha, \beta} u_{i}^{\alpha} u_{i}^{\beta} \mathcal{V} \nabla_{e_{\beta}} e_{\alpha}$, which, by the definition of the tensor $A$ and its skew-symmetricity, is just

$$
\mathcal{V} \tau(u)=\sum_{i, \alpha, \beta} u_{i}^{\alpha} u_{i}^{\beta} A\left(e_{\alpha}, e_{\beta}\right)=\sum_{i} A\left(\mathrm{~d} u\left(e_{i}\right), \mathrm{d} u\left(e_{i}\right)\right)=0 .
$$

This completes the proof of the lemma.

Lemma 1 tells us that the solution $u(\cdot, t)$ to $(*)$ is horizontal for any $t \in[0, T)$, so $\mathcal{V} \tau(u(\cdot, t))=0$ for $t \in[0, T)$. Thus, by the previous computation, we actually obtain

$$
\begin{aligned}
\left(\Delta-\frac{\partial}{\partial t}\right) e(u)= & |\nabla \mathrm{d} u|^{2}+<\operatorname{Ric}^{M}\left(\mathrm{~d} u\left(e_{i}\right), \mathrm{d} u\left(e_{i}\right)\right)> \\
& -<R^{X}\left(\mathrm{~d} u\left(e_{i}\right), \mathrm{d} u\left(e_{j}\right)\right) \mathrm{d} u\left(e_{i}\right), \mathrm{d} u\left(e_{j}\right)>.
\end{aligned}
$$

By the assumption on $\pi: X \rightarrow B, X$ has non-positive sectional curvature in the horizontal direction, so we have

$$
\left(\Delta-\frac{\partial}{\partial t}\right) e(u) \geq c e(u)
$$

for some constant $c$, which only depends on $M$. Denote the total energy of $u(\cdot, t)$ by $E(u(\cdot, t))$ for $t \in[0, T)$, i.e. $E(u(\cdot, t))=\int_{M} e(u(\cdot, t)) \mathrm{d} x$. Then, one has

$$
\begin{aligned}
& \frac{d}{d t} E(u(\cdot, t))=\frac{d}{d t} \int_{M}<d u, d u>d x=\int_{M}<\nabla_{\frac{\partial}{\partial t}} d u, d u>d x \\
= & \int_{M}<\nabla \frac{\partial u}{\partial t}, d u>d x=-\int_{M}<\frac{\partial}{\partial t} u, \tau(u)>d x=-\int_{M}|\mathcal{H} \tau(u)|^{2} d x \leq 0 .
\end{aligned}
$$

Summing all the above up, we have

Lemma 3. Suppose $u(x, t)$ is a solution of $(*)$. Then for some constant $c$,

$$
\left(\Delta-\frac{\partial}{\partial t}\right) e(u) \geq c e(u)
$$

furthermore, the total energy $E(u(\cdot, t))$ is a decreaseing function of $t$.

Combining the above lemma with Lemma 2.3.1 in [5], one has

Lemma 4. Let $t>0,0<R<\min \left(i(M), \frac{\pi}{2 \Lambda}\right)$, where $i(M)$ is the injective radius of $M$ and $\Lambda^{2}$ is an upper bound for the sectional curvature of $M$. Then, for all $x \in M$,

$$
e(u)(x, t) \leq c\left(t R^{-m-2}+t^{-\frac{m}{2}}\right) \int_{M} e(g)(y) d y,
$$


where $m=\operatorname{dim} M$ and $c$ is some constant depending only on the geometry of $M$; and for any $t_{0}<t$, in particular $t_{0}=0$,

$$
e(u)(x, t) \leq c R^{-2} \sup _{x \in M} e(u)\left(y, t_{0}\right)
$$

In the following, we want to derive a stability lemma. Let $g(x, s)$ be a smooth horizontal family of smooth horizontal maps from $M$ to $X$ with parameter $s \in$ $\left[0, s_{0}\right]$, i.e. both $g(\cdot, s)$ for any $s \in\left[0, s_{0}\right]$ and $\frac{\partial g}{\partial s}$ being horizontal. Suppose that $u(x, t, s)$ is a family of solutions of $(*)$ with initial data $g(x, s)$ for $0 \leq s \leq s_{0}$. As pointed out before, $\frac{\partial u}{\partial t}$ is horizontal; using the same discussion as in Lemma 1, we now show that $\frac{\partial u}{\partial s}$ is also horizontal: Fixing $x \in M$ and $s_{1} \in\left[0, s_{0}\right]$, one can then consider $u(x, t, s)$ as a variant of the curve $u\left(x, t, s_{1}\right)$. Take arbitrarily a vertical tangent vector $V$ at $u\left(x, 0, s_{1}\right)$ and translate parallelly $V$ along the $t$-curve $u\left(x, t, s_{1}\right)$ and then the $s$-curves $u(x, t, s)$, still denoted by $V$. (Note that $V$ is not necessarily parallel along other $t$-curves $u(x, t, s)$ for $s \neq s_{1}$.) Compute $\frac{\partial}{\partial t}<\frac{\partial u}{\partial s}, V>$

$$
\frac{\partial}{\partial t}<\frac{\partial u}{\partial s}, V>=<\nabla_{\frac{\partial}{\partial t}} \frac{\partial u}{\partial s}, V>=<\nabla_{\frac{\partial}{\partial s}} \frac{\partial u}{\partial t}, V>=\frac{\partial}{\partial s}<\frac{\partial u}{\partial t}, V>=0
$$

on the other hand, $\left.\left\langle\frac{\partial u}{\partial s}, V\right\rangle\right|_{t=0}=0$, therefore $\left\langle\frac{\partial u}{\partial s}, V\right\rangle=0$. Thus, for any fixed $t \in[0, T)$ and $s \in\left[0, s_{0}\right]$, the derivative of $u$ with respect to $s, \frac{\partial u}{\partial s}(\cdot, t, s)$, can be considered as a horizontal vector field of $X$ (if necessary, it needs to be considered as some section of certain pull-back bundle). Using the horizontality of $\frac{\partial u}{\partial s}(\cdot, t, s)$, we then have

Lemma 5. For every $s \in\left[0, s_{0}\right]$, the quantity

$$
\sup _{x \in M}\left|\frac{\partial u}{\partial s}\right|^{2}(x, t, s)
$$

is decreasing in $t$. Hence also the quantity

$$
\sup _{x \in M, s \in\left[0, s_{0}\right]}\left|\frac{\partial u}{\partial s}\right|^{2}(x, t, s)
$$

is a decreasing function of $t$.

Proof. As before, one can compute under a normal frame $\left\{e_{i}\right\}$

$$
\begin{aligned}
& \left(\Delta-\frac{\partial}{\partial t}\right)\left|\frac{\partial u}{\partial s}\right|^{2} \\
= & 2\left|\nabla \frac{\partial u}{\partial s}\right|^{2}-2 \sum_{i}<R\left(\frac{\partial u}{\partial s}, \mathrm{~d} u\left(e_{i}\right)\right) \frac{\partial u}{\partial s}, \mathrm{~d} u\left(e_{i}\right)>.
\end{aligned}
$$

Here we use the heat eqaution and the horizontality of $\frac{\partial u}{\partial s}$. Thus, by the assumption for the sectional curvature in the horizontal direction, we have

$$
\left(\Delta-\frac{\partial}{\partial t}\right)\left|\frac{\partial u}{\partial s}\right|^{2} \geq 0
$$

The lemma then follows from the maximum principle for parabolic equations. 
In order to apply the regularity theorems for elliptic equations, we have to make sure that the solution of $(*)$ with the given initial data $g$ lies in a suitable coordinate chart of $X$ when the domain considered is enough small and the time interval short enough. We have obtained a point-wise upper bound of the derivatives of $u$ with respect to the space variables, so we still have to derive a bound for the time derivative of the solution. This can be done by applying the above lemma.

Lemma 6. Suppose that $u(x, t)$ is a solution of $(*)$ with the initial data $g$ for $t \in[0, T)$. Then for all $t \in[0, T)$ and $x \in M$

$$
\left|\frac{\partial u(x, t)}{\partial t}\right| \leq \sup _{y \in M}\left|\frac{\partial u(y, o)}{\partial t}\right| .
$$

Proof. Setting $u(x, t, s)=u(x, t+s)$, then $u(x, t, s)$ can be considered as a family of solutions to $(*)$ with a family of initial data $u(x, s)$. Applying the preceeding lemma to $u(x, t, s)$, we then get the present lemma.

Fix $x \in M$ and $t \in[0, T)$. As before, we take a normal frame $\left\{e_{i}\right\}$ at $x$, and a orthogonal frame $\left\{e_{\alpha}, e_{\beta}, e_{\gamma}, \cdots, e_{\mu}, e_{\nu}, \cdots\right\}$ at $u(x, t)$ with the property that $\left\{e_{\alpha}, \cdots\right\}$ are horizontal and $\left\{e_{\mu}, \cdots\right\}$ are vertical; as pointed out before, under such a restriction, one cannot get a normal frame at $u(x, t)$ in general. Then, the heat equation $(*)$ can be rewritten under such frames at $(x, t)$ as

$$
\left(*^{\prime}\right) \sum_{i} u_{i i}^{\alpha}+\sum_{i, \beta, \gamma} \Gamma_{\beta \gamma}^{\alpha} u_{i}^{\beta} u_{i}^{\gamma}=\frac{\partial u^{\alpha}}{\partial t} .
$$

Remark. Note that the solution with the initial data $g$ is horizontal for both the space variable and the time variable, as seen in Lemma 1. So by Lemma 2, $\mathcal{V} \tau(u)=0$, i.e. $\mathcal{H} \tau(u)=\tau(u)$. Thus we can actually omit $\mathcal{H}$ in the equation $(*)$ and think that $u$ just satisfies the usual heat equation for harmonic maps $\tau(u)-\frac{\partial u}{\partial t}=0$. In the following estimate, we will actually adopt this point of view although it will not be pointed out explicitly.

Lemma 7. Suppose that $u(x, t)$ is a solution of $(*)\left(\right.$ or $\left.\left(*^{\prime}\right)\right)$ with the initial data $g$ for $t \in[0, T)$. Then for every $\alpha \in(0,1)$

$$
\|u(\cdot, t)\|_{C^{2+\alpha}(M ; X)}+\left\|\frac{\partial u}{\partial t}(\cdot, t)\right\|_{C^{\alpha}(M ; X)} \leq c,
$$

where $c$ depends on $\alpha$, the initial data $g(x)$, and the geometry of $M$ and $X$, but not on $t$.

Proof. Rewrite $\left(*^{\prime}\right)$ as

$$
\sum_{i} u_{i i}^{\alpha}=-\sum_{i, \beta, \gamma} \Gamma_{\beta \gamma}^{\alpha} u_{i}^{\beta} u_{i}^{\gamma}+\frac{\partial u^{\alpha}}{\partial t} .
$$

If we restrict the solution $u$ to a suitable small coordinate chart at the point $x_{0} \in M$, say $B\left(x_{0}, \rho\right)$ with $\rho$ enough small, and a suitable small time interval 
$\left[t_{0}, t_{1}\right], u(x, t)$ will stay in a certain coordinate chart of $X$ by the lemma 4 and the lemma 6 ; moreover, the two lemmata also imply that the right-hand side of the above equation is bounded (note that the bound does not depend on $t$ ), this, by elliptic regularity theory, then implies a bound (again not depending on $t$ ) for $\|u(\cdot, t)\|_{C^{1+\alpha}(M ; X)}$ on a smaller coordinate chart, say $B\left(x_{0}, \frac{\rho}{2}\right)$ (see [5], Theorem 2.2.1). Thus, the right-hand side of the following parabolic equation

$$
\frac{\partial u^{\alpha}}{\partial t}-\sum_{i} u_{i i}^{\alpha}=\sum_{i, \beta, \gamma} \Gamma_{\beta \gamma}^{\alpha} u_{i}^{\beta} u_{i}^{\gamma}
$$

is bounded (the bound being independent of $t$ ) in $C^{\alpha}(M ; X)$, and hence the Schauder estimate for parabolic equations then implies the estimate in the lemma, at least in the above small coordinate chart; but $M$ is compact, so the estimate is valid on $M$.

Based on the local existence of solutions and the above Schauder estimate, one has the following global existence theorem for $(*)$ with the initial data $g$.

Theorem 1. The solution $u(x, t)$ of the heat equation $(*)$ with the horizontal initial data $g$ exists for all $t \in[0, \infty)$, provided that the Riemannian submersion $\pi: X \rightarrow B$ satisfies the Chow condition and that $B$ has non-positive sectional curvature.

In the following, we will show that the global solution $u(\cdot, t)$ in the theorem above converges to a horizontal harmonic map as $t$ goes to infinity. As seen before, we have shown the energy decay formula, namely

$$
\frac{\mathrm{d}}{\mathrm{d} t} E(u(\cdot, t))=-\int_{M}\left|\frac{\partial u(x, t)}{\partial t}\right|^{2} \mathrm{~d} x=-\int_{M}|\mathcal{H} \tau(u)|^{2} \mathrm{~d} x
$$

observe also that the energy function $E(u(\cdot, t))$ in $t$ is nonnegative for $t \in$ $[0, \infty)$, so there exists a sequence $\left\{t_{n}\right\}_{n=1}^{\infty}$ with $t_{n} \rightarrow \infty$ as $n \rightarrow \infty$ satisfying $\left.\frac{\mathrm{d}}{\mathrm{d} t} E(u(\cdot, t))\right|_{t_{n}} \rightarrow 0$ as $n \rightarrow \infty$, this is just equivalent to saying

$$
\int_{M}\left|\frac{\partial u}{\partial t}\left(x, t_{n}\right)\right|^{2} \mathrm{~d} x \rightarrow 0, \text { as } n \rightarrow \infty .
$$

On the other hand, as seen in Lemma $7, \frac{\partial u}{\partial t}(\cdot, t)$ has a $C^{\alpha}$-bound independent of the time $t$, so we obtain

Lemma 8. There exists a sequence $\left\{t_{n}\right\}_{n=1}^{\infty}$ with $t_{n} \rightarrow \infty$ as $n \rightarrow \infty$, for which $\frac{\partial u}{\partial t}\left(x, t_{n}\right)$ converges to zero uniformly in $x \in M$ as $n \rightarrow \infty$.

Lemma 7 also tells us that $u(\cdot, t)$ has a time-independent $C^{2+\alpha}$-bound, so one obtains, by possibly passing to a subsequence of $\left\{t_{n}\right\}$, that $u\left(\cdot, t_{n}\right)$ converges at least $C^{2}$-uniformly to a map $u: M \rightarrow X$, which is moreover horizontal; furthermore, since $\left\{u\left(\cdot, t_{n}\right)\right\}$ is at least $C^{2}$-uniformly convergent to $u$ and both $u\left(\cdot, t_{n}\right)$ and $u$ are horizontal, so by the Hopf-Rinow theorem, as mentioned in the Introduction, some $u\left(\cdot, t_{n}\right)$, and hence $g(\cdot)$, is homotopic to $u(\cdot)$ by a certain horizontal homotopy $h(\cdot, s)$ for $s \in[0,1]$ with $h(\cdot, 0)=u\left(\cdot, t_{n}\right)$ and $h(\cdot, 1)=u(\cdot)$. 
Here by the homotopy $h(\cdot, s)$ being horizontal we mean that $h(\cdot, s)$ for each $s \in[0,1]$ is a horizontal map and the $s$-curves are also horizontal. Again since $\left\{u\left(\cdot, t_{n}\right)\right\}$ uniformly converges to $u$, w. l. o. g., we can assume that the length of the $s$-curves $h(x, s)$ have a sufficient small upper bound $\epsilon>0$ independent of $x \in M$. We now observe the family of the solutions $u^{\prime}(x, t, s)(s \in[0,1])$ to $(*)$ with $h(x, s)$ being the family of initial maps. It is clear that $u^{\prime}(x, t, 1)=u(x)$ since $\mathcal{H} \tau(u)=0$ and $h(x, 1)=u(x)$; while $u^{\prime}(x, t, 0)=u\left(x, t+t_{n}\right)$. By the Lemma 5 , the supremum with respect to $x$ of the length of $s$-curves $u^{\prime}(\cdot, t, s)$ is a decreasing function of $t$ and hence less than $\epsilon$. Since $\epsilon$ is arbitrary, we have that $u(x, t)$ converges uniformly to $u(x)$ in $t$ in the sense of $C^{0}$, not only for a subsequence $\left\{t_{n}\right\}$. Applying this to the heat equation $(*)$, one obtains

$$
\mathcal{H} \tau(u)=0 .
$$

Finally, the horizontality of $u(x)$ and the Lemma 2 tell us that $\mathcal{V} \tau(u)=0$, and hence

$$
\tau(u)=0,
$$

i.e. the limit $u$ is a horizontal harmonic map. Thus we have

Theorem 2. Suppose that $\pi: X \rightarrow B$ is a Riemannian submersion satisfying the Chow conditions and that $B$ has non-positive sectional curvature. Let $M$ be a compact Riemannian manifold and $g: M \rightarrow X$ a horizontal smooth map from $M$ to $X$. Then there exists a horizontal harmonic map $u: M \rightarrow X$ from $M$ into $X$, which is homotopic to $g$ by a horizontal homotopy.

Remark. The theorem above is actually valid in a more general setting, namely the equivariant one: Let $\phi: \pi_{1}(M) \rightarrow \pi_{1}(X)$ be a homomorphism and $g$ a $\phi$-equivariant map from $M$ into $X$, then one can solve the corresponding heat equation $(*)$ and obtain similar results, e.g. the existence for $\phi$-equivariant horizontal harmonic maps. We omit this, but point out that in our future applications we shall use this setting. We shall come back to this in [6].

\section{Acknowlegements}

We want to thank Guofang Wang for valuable discussions. The main part of this work was completed when the second author was visiting the Max Planck Institute for Mathematics in the Sciences. He would like to thank the institute for hospitality and good working conditions.

\section{References}

[1] A. Bellaïche, The tangent space in sub-Riemannian geometry, in: Sub-Riemannian Geoemtry, Progress in Mathematics, Birkhäuser (1996) 1-78.

[2] A. Besse, Einstein Manifolds, Ergebnisse der Mathematik und ihrer Grenzgebiete 3. Folge Band 10, Springer-verlag, (1987).

[3] P. Griffiths and W. Schmid, Locally homogeneous complex manifolds, Acta Mathematica 123 (1969) 253-302.

[4] S. Helgason, Differential Geometry and Symmetric Spaces, New York, Academic Press (1962). 
[5] J. Jost, Nonlinear Methods in Riemannian and Kählerian Geometry, DMV Seminar, Band 10, Birkhäuser (1988).

[6] J. Jost and Y.-H. Yang, Horizontal harmonic maps into locally homogeneous complex manifolds, in preparation.

[7] S. Kobayashi and K. Nomizu, Foundations of Differential Geometry, Vol.II, Interscience Publishers (1969).

[8] B. O'Neill, The fundamental equations of a submersion, Michigan Mathematical Journal, 13 (1966) 459-469.

[9] J.-P. Serre, Complex Semisimple Lie Algebras, Springer-Verlag, New York, (1987).

[10] C. Simpson, Higgs bundles and Local systems, Inst. Hautes Études Sci. Publ. Math. 75 (1992) 5-95.

MaX-Planck-Institute for Mathematics in the Sciences, Inselstrasse 22, D-04103 LeIPZIG, Germany

E-mail address: juergen.jost@mis.mpg.de

Department of Applied Mathematics, Tongui University, Shanghai 200092, China, E-mail address: yhyang@mail.tongji.edu.cn 